$\begin{array}{cc}\text { Programa de Pós-Graduação em Engenharia de Produção - PPGEP } & \\ \text { Laboratório de Qualidade de Vida - LaQVida } & \\ \text { Universidade Tecnológica Federal do Paraná - UTFPR } & \text { REVISTA BRASILEIRA DE } \\ \text { Ponta Grossa - PR - Brasil } & \text { QUALIDADE DE VIDA }\end{array}$

\title{
Percepção de qualidade de vida entre idosos jovens e longevos praticantes de hidroginástica
}

\section{Perception of quality of life of elderly among young elderly and oldest practicing gymnastics}

\author{
Priscila Mari dos Santos \\ Universidade do Estado de Santa Catarina - UDESC - Florianópolis - Brasil \\ priscilamarisantos@hotmail.com \\ Bruna Carli de Souza \\ Universidade do Estado de Santa Catarina - UDESC - Florianópolis - Brasil \\ brunenha_90@hotmail.com \\ Alcyane Marinho \\ Universidade do Estado de Santa Catarina - UDESC - Florianópolis - Brasil \\ alcyane.marinho@udesc.br \\ Giovana Zarpellon Mazo \\ Universidade do Estado de Santa Catarina - UDESC - Florianópolis - Brasil \\ giovana.mazo@udesc.br
}

\section{RESUMO}

OBJETIVO: Verificar a relação da percepção de qualidade de vida - QV entre idosos jovens e longevos praticantes de hidroginástica.

MÉTODOS: Participaram do estudo 76 idosos, sendo 70 na categoria de idosos jovens (entre 60 e 79 anos) e seis na categoria de idosos longevos (80 anos ou mais). Os questionários de QV da Organização Mundial da Saúde (WHOQOL-BREF e WHOQOL-OLD) foram aplicados aos idosos em forma de entrevista individual. A análise dos dados foi realizada por meio da estatística descritiva e do Teste U de Mann-Whitney, com um nível de significância de 5\%.

RESULTADOS: Os idosos apresentaram média de idade de 70,9 $\pm 6,3$ anos, sendo que 92,1\% (70) pertencem ao sexo feminino. Constatou-se que os idosos têm uma boa percepção de QV, influenciada em ordem decrescente pelo domínio das relações sociais $(74,3 \pm 16,2)$, do meio ambiente $(71,2 \pm 11,9)$, psicológico $(66,6 \pm 9)$ e físico $(60,7 \pm 11,2)$. Na avaliação específica, a percepção de QV também foi considerada boa, influenciada pelas facetas da participação social $(73,1 \pm 13)$, intimidade $(72,5 \pm 17,2)$, atividades passadas, presentes e futuras $(71,9 \pm 14,4)$, autonomia $(71,1 \pm 14,9)$, funcionamento dos sentidos $(70,2 \pm 18,2)$ e morte e morrer $(67,7 \pm 22,5)$. No entanto, na comparação entre os grupos foram observadas diferenças estatisticamente significativas nos domínios referentes aos aspectos físicos $(p=0,047)$ e das relações sociais $(p=0,047)$. Em ambos os domínios, a média foi significativamente maior no grupo de idosos jovens. Na avaliação de QV específica para idosos foram encontradas diferenças estatísticas nas facetas 'morte e morrer' $(\mathrm{p}=0,037)$ e 'intimidade' $(\mathrm{p}=0,049)$. No caso da primeira faceta, o grupo de idosos longevos apresentou média significativamente maior, e no caso da segunda, este grupo apresentou média significativamente menor. 
CONCLUSÕES: Apesar de os idosos jovens alcançarem maior média que os longevos nos domínios físico e das relações sociais e na faceta da intimidade, ambos os grupos têm boa percepção de QV.

PALAVRAS-CHAVE: Idosos. Qualidade de vida. Hidroginástica.

\section{ABSTRACT}

OBJECTIVE: To investigate the relationship perceived of quality of life - QOL among young elderly and oldest practicing gymnastics.

METHODS: The study included 76 elderly, 70 in the category of young elderly (among 60 and 79 years) and six in the category of the oldest old (80 and over). Questionnaires WHOQOL-BREF and WHOQOL-OLD were applied to the elderly in individual interviews. Data analysis was performed using the statistical descriptive and Mann-Whitney U test, with significance level of 5\%.

RESULTS: The elderly had a mean age of $70.9 \pm 6.3$ years, and $92,1 \%$ (70) were female. It was found that the elderly have a good perception of QOL, influenced in descending order by the field of social relations $(74.3 \pm 16.2)$, environment $(71.2 \pm 11.9)$, psychological $(66.6 \pm 9)$ and physical $(60.7 \pm 11.2)$. The perception of QOL was also considered good, influenced respectively by facets social participation $(73.1 \pm 13)$, intimacy $(72.5 \pm 17.2)$, activities past, present and future (71.9 \pm 14.4$)$, autonomy (71.1 \pm 14.9$)$, operation of the senses $(70.2 \pm 18.2)$, and death and dying $(67.7 \pm 22.5)$. However, the comparison between the groups were statistically significant differences in the areas related to physical aspects $(\mathrm{p}=0.047)$ and social relations $(\mathrm{p}=0.047)$. In both areas, the average was significantly higher in the elderly group youth. In the assessment of QOL specific to seniors were statistical differences in the facets "death and dying" $(p=0.037)$ and "intimacy" $(p=0.049)$. For the first aspect, the group of oldest old had significantly higher average, and in the second case, this group had significantly lower average.

CONCLUSIONS: Although older youth achieve higher average than the oldest in the physical and social relations and facet of intimacy, both groups have good perception of QOL.

KEYWORDS: Elderly. Quality of life. Gymnastics.

\section{Introdução}

Nos últimos anos, no Brasil, pôde-se observar o desenvolvimento sistemático de um processo de envelhecimento populacional. Atualmente, já existem mais de 20 milhões de idosos no país, correspondendo a $10,8 \%$ da população total e, estima-se que no ano de 2050, o número de idosos no Brasil ultrapasse os 64 milhões, representando mais de 22\% da população (OMS, 2005; IBGE, 2010, 2011).

Este processo de envelhecimento populacional é resultado, principalmente, das tendências históricas de redução das taxas de fecundidade e de mortalidade, e do aumento da esperança de vida ao nascer (MAZO; LOPES; BENEDETTI, 2009). No ano de 2009, a esperança de vida já ultrapassava os 73,1 anos de idade entre os brasileiros (IBGE, 2010) e, no ano de 2050, espera-se que esse indicador alcance o patamar de 81,3 anos (IBGE, 2008).

Diante deste cenário, Fleck et al. (2008) ressaltam que descobrir virtudes na velhice, prolongar a juventude e envelhecer com boa percepção de qualidade de vida - QV, têm sido preocupações sistemáticas do ser humano, manifestadas em diversas áreas do conhecimento. Os autores salientam que, nesse contexto, passou a ser importante dispor de maneiras de mensurar a forma como as pessoas estão alcançado e vivendo esses anos a mais de suas vidas, ou seja, de medir a percepção de QV dos idosos. 
De acordo com o Grupo de QV da Organização Mundial da Saúde - WHOQOL GROUP (1995, p. 1405), a QV pode ser entendida como "[...] a percepção do indivíduo de sua posição na vida, no contexto da cultura e sistemas de valores nos quais ele vive e em relação aos seus objetivos, expectativas, padrões e preocupações". Nessa perspectiva, Fleck et al. (2008) ressaltam que esta concepção de QV se refere a uma percepção subjetiva sobre a vida de cada indivíduo, influenciada pelo contexto cultural, social e ambiental que o mesmo está inserido.

Considerando que a avaliação da QV envolve aspectos subjetivos, os determinantes de uma boa percepção de QV variam de indivíduo para indivíduo, inclusive entre grupos específicos de idade. O envelhecimento é uma experiência singular e, em virtude de seus valores e histórias de vida, os idosos diferem dos jovens e também entre si, nos extremos de idade (JOIA; RUIZ; DONALISIO, 2007).

A Organização Mundial da Saúde - OMS considera idosos os indivíduos com 60 anos ou mais. Porém, no Brasil, de acordo com o Instituto de Pesquisa Econômica Aplicada - IPEA (2008), os indivíduos que têm entre 60 e 69 anos são considerados idosos jovens; aqueles que têm de 70 a 79 anos são medianamente idosos; e os com 80 anos ou mais são considerados muito idosos, ou longevos. Nesse contexto, surgem algumas indagações: idosos jovens têm melhor percepção de QV que idosos longevos? Em que aspectos estes indivíduos diferem na avaliação da QV?

Nessa direção, Mazo (2008) salienta o interesse de diversas áreas do conhecimento (tais como Gerontologia, Psicologia, Educação Física, entre outras) de investigar a percepção de QV de idosos de distintos grupos de idade, no sentido de compreender as condições que permitem uma boa percepção de QV na velhice, as mudanças que ocorrem com o avanço da idade, entre outros fatores que contribuam com subsídios para a efetivação de políticas públicas e intervenções sociais a favor desta população.

No contexto da área da Educação Física, observa-se o interesse por parte dos pesquisadores em investigar idosos praticantes de exercícios físicos, uma vez que estes estão sendo apontados por estudiosos como essenciais para a boa percepção de QV entre idosos, especialmente por seus efeitos positivos, amplamente reconhecidos, sobre a capacidade funcional, a saúde, o bem-estar psicológico, a sociabilidade, a vitalidade, a independência e a autonomia (TOSCANO; OLIVEIRA, 2009; TRIBESS; VIRTUOSO JÚNIOR; OLIVEIRA, 2012; UENO et al., 2012).

Dentre as diversas possibilidades de exercícios físicos disponíveis para os idosos, a hidroginástica vem se destacando pela conquista cada vez maior de adeptos (SIMÕES; PORTES JÚNIOR; MOREIRA, 2011). Estudos apontam que a prática regular de hidroginástica é adequada à população idosa, em função do ambiente aquático facilitar a locomoção, oferecendo menos impacto aos ossos e às articulações destes indivíduos que, naturalmente, estão mais frágeis em virtude do avanço da idade (ALVES et al., 2004; CERRI; SIMÕES, 2007).

Além de ser amplamente recomendada pelos médicos, a hidroginástica entre idosos também é procurada pelos próprios benefícios gerais da prática de exercício físico, pela busca de uma vida saudável e ativa, e também pela sociabilidade (MAZO; CARDOSO; AGUILAR, 2006). Conforme apontam Cerri e Simões (2007), por ser um exercício desenvolvido em grupo é possível inserir nas aulas diversos tipos de atividades que promovam o lúdico, a socialização e a interação entre os participantes, concomitantemente com a melhora da aptidão física geral relacionada à saúde.

Nesse contexto, é possível observar que diferentes programas de exercício físico oferecem a prática da hidroginástica, especialmente para idosos de distintos grupos de idade. Assim, percebe-se a premência em investigar a percepção de QV de idosos praticantes de hidroginástica. Portanto, o presente estudo teve como objetivo verificar a relação da percepção de QV entre idosos jovens e longevos praticantes de hidroginástica.

\section{Metodologia}

O presente estudo, de corte transversal, aprovado pelo Comitê de Ética em Pesquisa com Seres Humanos da Universidade do Estado de Santa Catarina - UDESC (número de protocolo 
98/2011), foi realizado por meio de uma investigação descritiva com abordagem quantitativa dos dados.

A população foi constituída por 210 idosos, de ambos os sexos, participantes do programa de extensão universitária Grupo de Estudos da Terceira Idade - GETI e a amostragem por conveniência foi realizada com todos os presentes na última semana de atividades deste grupo em 2011. Criado em 1989 como um programa pioneiro em Florianópolis (SC), o GETI é, hoje, um programa consolidado no atendimento aos idosos, desenvolvido no Centro de Ciências da Saúde e do Esporte - CEFID da UDESC.

Assim, compuserem a amostra deste estudo 76 idosos praticantes de hidroginástica no GETI, com média de idade de 70,9+6,3 anos, sendo $92,1 \%$ do sexo feminino. As aulas desta modalidade são ministradas duas vezes por semana, com duração de 50 minutos e intensidade moderada. Foram considerados idosos os indivíduos com 60 anos ou mais, de acordo com o estabelecido pela OMS (2005). Porém, os participantes foram divididos entre a categoria de idosos jovens (entre 60 e 79 anos) e a categoria de idosos longevos (80 anos ou mais), de acordo com as categorias gerais de idade estabelecidas pelo IPEA (2008). A primeira categoria foi ocupada por $92,1 \%$ (70) dos idosos e a segunda por 7,9\% (6). Todos os idosos aceitaram participar voluntariamente do estudo, assinando um termo de consentimento livre e esclarecido.

Para a coleta de dados foram utilizados questionários autoaplicáveis, respondidos de forma presencial, mas administrados pelos pesquisadores deste estudo (em virtude dos problemas visuais, das dificuldades de leitura e de alguns idosos serem analfabetos), a saber:

a) WHOQOL-BREF - Questionário de Qualidade de Vida da Organização Mundial da Saúde: constituído por 26 questões, como uma forma geral e reduzida das 100 questões originais. Entre estas, 24 abrangem quatro domínios para a avaliação da QV, nomeadamente os domínios físico, psicológico, das relações sociais e o do meio ambiente. As outras duas questões avaliam de forma mais geral a QV global e a percepção de saúde. Os escores dos domínios são calculados por meio da soma dos escores da média das ' $n$ ' questões que compõem cada domínio. O resultado é multiplicado por quatro, sendo representado em uma escala de 4 a 20, a qual, por sua vez, é convertida para uma escala de 0 a 100 . Quanto mais próximo de 100, mais positiva é a percepção da QV do indivíduo (WHOQOL GROUP, 1998). Fleck et al. (2000) realizaram o teste de campo brasileiro deste instrumento com uma amostra de 300 indivíduos de Porto Alegre (RS), observando características satisfatórias de consistência interna, validade e fidedignidade teste-reteste;

b) WHOQOL-OLD - Questionário de Qualidade de Vida da Organização Mundial da Saúde para Idosos: desenvolvido para complementar a avaliação geral (por meio do WHOQOL-BREF) é constituído por 24 questões, divididas em seis 'facetas' (funcionamento dos sentidos - FS; autonomia - AUT; atividades passadas, presentes e futuras - PPF; participação social - PSO; morte e morrer - MEM; e intimidade - INT). Cada faceta é composta por quatro itens, gerando escores que variam de 4 a 20, que podem ser expressos em porcentagem, variando de 0 a 100. Quanto mais próximo de 100, mais positiva é a percepção da QV dos idosos (FLECK et al., 2008). Este instrumento foi desenvolvido e validado para o português brasileiro por Fleck, Chachamovich e Trentini (2006), com a aplicação em uma amostra de 424 idosos de Porto Alegre (RS).

As informações coletadas foram analisadas com a utilização do programa estatístico SPSS versão 17.0, realizando-se, inicialmente, o teste de Kolmogorov-Smirnov, a fim de verificar a normalidade dos dados. Uma vez que estes não apresentaram uma distribuição normal, foi aplicada a estatística inferencial não paramétrica, por meio do Teste U de Mann-Whitney. Adotou-se o nível de significância de 5\%. Também foi aplicada a estatística descritiva (frequência simples, porcentagem, média e desvio padrão).

\section{Resultados}

Os 76 idosos investigados neste estudo apresentaram média de idade de 70,9 $\pm 6,3$ anos. A menor idade encontrada foi 60 anos e a maior 87 anos. Compuseram o grupo de idosos longevos, seis idosos com 80 anos ou mais, sendo cinco do sexo feminino e um do sexo masculino. O grupo 
de idosos jovens foi composto por 70 idosos com idades entre 60 e 79 anos, sendo 65 do sexo feminino e 5 do sexo masculino. No grupo total de idosos, identificou-se $92,1 \%$ (70) pertencentes ao sexo feminino.

Na Tabela 1 estão apresentadas as médias dos escores dos domínios de avaliação da QV, por meio do WHOQOL-BREF, entre o grupo de idosos jovens e o grupo de idosos longevos, assim como o escore do teste U de Mann-Whitney e o valor de p.

Tabela 1 - Associação entre os domínios de avaliação da QV (WHOQOL-BREF) entre o grupo de idosos jovens e idosos longevos

\begin{tabular}{|c|c|c|c|c|c|}
\hline $\begin{array}{c}\text { Domínios } \\
\text { WHOQOL-BREF }\end{array}$ & $\begin{array}{c}\begin{array}{c}\text { Idosos jovens } \\
(\text { média } \pm)\end{array} \\
\end{array}$ & $\begin{array}{c}\text { Idosos longevos } \\
\text { (média } \pm \text { ) }\end{array}$ & $\begin{array}{c}\text { Total } \\
(\text { média } \pm)\end{array}$ & Valor de $\mathbf{U}$ & Valor de $p$ \\
\hline Físico & $61,5 \pm 10,9$ & $52,5 \pm 10,9$ & $60,7 \pm 11,2$ & 107,5 & $0,047 *$ \\
\hline Psicológico & $66,8 \pm 9,2$ & $64,9 \pm 7,2$ & $66,6 \pm 9$ & 173 & 0,471 \\
\hline Relações sociais & $75,2 \pm 16,4$ & $65,5 \pm 10,1$ & $74,3 \pm 16,2$ & 108,5 & $0,047 *$ \\
\hline Meio ambiente & $71,8 \pm 12,1$ & $63,4 \pm 7,4$ & $71,2 \pm 11,9$ & 132 & 0,131 \\
\hline Avaliação global & $72,9 \pm 13,6$ & $67,9 \pm 12,2$ & $72,4 \pm 13,7$ & 162 & 0,323 \\
\hline
\end{tabular}

$+=$ desvio padrão. $* \mathrm{p}<0,05$.

Fonte: Autoria própria (2011).

Observa-se que há diferenças estatisticamente significativas entre a percepção de QV de idosos jovens e de idosos longevos nos domínios referentes aos aspectos físicos $(\mathrm{p}=0,047)$ e das relações sociais $(p=0,047)$. Em ambos os domínios, a média do escore de avaliação foi significativamente maior no grupo de idosos jovens. Nos domínios psicológicos e do meio ambiente, e no item que avalia a QV e a percepção de saúde de forma global, a média dos escores foi ligeiramente maior também no grupo de idosos jovens, mas nestes casos não foram constatadas diferenças estatisticamente significativas entre os grupos.

Percebe-se que independentemente da comparação estatística entre os grupos, os idosos participantes do estudo têm uma percepção relativamente boa de $\mathrm{QV}$, uma vez que as médias dos escores dos domínios ultrapassaram o ponto médio da escala de avaliação, situada entre os extremos de 0 e 100. No contexto total, o domínio que contribui com a maior média foi o das relações sociais $(74,3 \pm 16,2)$, seguido pelo domínio do meio ambiente $(71,2 \pm 11,9)$, psicológico $(66,6 \pm 9)$ e físico $(60,7 \pm 11,2)$. No grupo de idosos jovens também foi observada esta ordem de contribuição. Porém, no grupo de idosos longevos foi verificado que o domínio das relações sociais também apresentou a maior média $(65,5 \pm 10,1)$, mas na sequência a média encontrada no domínio psicológico foi $(64,9 \pm 7,2)$ e no do meio ambiente $(63,4 \pm 7,4)$. O domínio físico também apresentou a menor média neste grupo $(52,5 \pm 10,9)$.

Na Tabela 2 estão listados os escores das facetas de avaliação da QV, por meio do WHOQOL-OLD, entre o grupo de idosos jovens e o grupo de idosos longevos, assim como o escore do teste U de Mann-Whitney e o valor de $\mathrm{p}$. 
Tabela 2 - Associação entre as facetas de avaliação da QV (WHOQOL-OLD) entre o grupo de idosos jovens e idosos longevos

\begin{tabular}{lccccc}
\hline $\begin{array}{c}\text { Facetas } \\
\text { WHOQOL-OLD }\end{array}$ & $\begin{array}{c}\text { Idosos jovens } \\
(\mathbf{m e ́ d i a} \pm)\end{array}$ & $\begin{array}{c}\text { Idosos longevos } \\
(\mathbf{m e ́ d i a} \pm)\end{array}$ & $\begin{array}{c}\text { Total }(\mathbf{m e ́ d i a} \\
\mathbf{n})\end{array}$ & Valor de U & Valor de p \\
\hline FS & $70,3 \pm 18,6$ & $69,8 \pm 13,9$ & $70,2 \pm 18,2$ & 200,5 & 0,854 \\
AUT & $71,1 \pm 15,3$ & $71,9 \pm 10,3$ & $71,1 \pm 14,9$ & 207,5 & 0,961 \\
PPF & $72,5 \pm 14,8$ & $64,6 \pm 6,5$ & $71,9 \pm 14,4$ & 164 & 0,369 \\
PSO & $73,5 \pm 13,3$ & $68,8 \pm 8,8$ & $73,1 \pm 13$ & 195 & 0,768 \\
MEM & $66,5 \pm 22,6$ & $82,3 \pm 17,2$ & $67,7 \pm 22,5$ & 174,5 & $0,037^{*}$ \\
INT & $74 \pm 16,9$ & $55,2 \pm 9,2$ & $72,5 \pm 17,2$ & 109 & $0,049^{*}$ \\
\hline
\end{tabular}

$\pm=$ desvio padrão. $* \mathrm{p}<0,05$. FS - funcionamento dos sentidos; AUT - autonomia; PPF - atividades passadas, presentes e futuras; PSO - participação social; MEM - morte e morrer; INT - intimidade.

Fonte: Autoria própria (2011).

Entre os dois grupos de idosos verificaram-se diferenças estatisticamente significativas nas facetas de QV MEM ( $\mathrm{p}=0,037)$ e INT $(\mathrm{p}=0,049)$. No caso da primeira faceta, o grupo de idosos longevos apresentou maior média $(82,3 \pm 17,2)$; e no caso da segunda, este grupo apresentou média inferior $(55,2 \pm 9,2)$ ao do grupo de idosos jovens $(74 \pm 16,9)$.

Esta avaliação específica para idosos confirmou os resultados da avaliação geral, no sentido de apresentar médias relativamente altas entre as facetas, revelando a boa percepção de QV de todos os idosos. As facetas que contribuíram para essa avaliação positiva com as maiores médias foram: PSO $(73,1 \pm 13)$, INT $(72,5 \pm 17,2)$, PPF $(71,9 \pm 14,4)$, AUT $(71,1 \pm 14,9)$, FS $(70,2 \pm 18,2)$ e MEM $(67,7 \pm 22,5)$.

No grupo de idosos jovens esta ordem de contribuição das facetas também foi constatada, alterando-se apenas a INT para a primeira posição com a maior média $(74+16,9)$ e a PSO para a segunda $(73,5 \pm 13,3)$. No grupo de idosos longevos a ordem das maiores médias se apresentou de forma bastante diferenciada: a faceta MEM ocupou a primeira posição $(82,3 \pm 17,2)$, seguida pela AUT $(71,9 \pm 10,3)$, FS $(69,8 \pm 13,9)$, PSO $(68,8 \pm 8,8)$, PPF $(64,6+6,5)$ e INT $(55,2 \pm 9,2)$.

\section{Discussões}

Uma das limitações desse estudo esteve relacionada à amostra pequena de idosos longevos. Sugere-se que futuros estudos investiguem as percepções de QV de idosos incluindo em suas análises esse grupo populacional de idosos e, de preferência, em maior quantidade. Isso não apenas contribuiria para a minimização da escassez de trabalhos com idosos em idades mais avançadas, como também possibilitaria a descoberta sobre os fatores determinantes de uma boa QV nesse período da vida. Estudos similares a este, como os de Pereira et al. (2006) e de Maués et al. (2010), incluíram idosos longevos em suas amostras, porém apenas o último analisou uma quantidade de idosos longevos similar a de idosos jovens.

Apesar destas limitações, observou-se neste estudo o alto percentual de idosos jovens, que pode ser explicado pelo fato de existir mais idosos com idades entre 60 e 79 anos na própria população brasileira como um todo. No entanto, o grupo de idosos longevos está aumentando nos últimos anos, indicando que a própria população idosa está envelhecendo, com características e diferenças que ultrapassam a simples composição etária (IBGE, 2011).

A heterogeneidade deste grupo populacional também pode ser percebida na composição por sexo da população. Há predominância feminina em todas as faixas etárias, haja vista o processo histórico de maior mortalidade masculina ao longo da vida, observado em todo o mundo. Na terceira idade a mortalidade diferencial entre os sexos faz com que as mulheres vivam mais que os homens, ficando viúvas mais cedo, alcançando esta fase da vida com mais frequência ou permanecendo nela por mais tempo que os homens (CAMARANO; KANSO; MELLO, 2004). 
Esse forte componente feminino do processo de envelhecimento repercute na maior participação de idosas no contexto de programas voltados à terceira idade. Diversos estudos que se propuseram a investigar grupos de idosos em diferentes contextos, constataram a expressiva participação de mulheres (MAUÉS et al., 2010; BECKERT; IRIGARAY; TRENTINI, 2012; UENO et al., 2012). Todavia, Camarano, Kanso e Mello (2004) salientam que essa predominância feminina também pode ser decorrente da característica das mulheres em geral, de participar mais que os homens de atividades extradomésticas, de grupos e organizações.

Diante deste contexto, deve-se refletir sobre as características, peculiaridades e diferenças que existem no próprio segmento populacional de idosos. De acordo com Maués et al. (2010), o aumento da longevidade traz ao profissional da saúde o desafio de conhecer os fatores relacionados à maior expectativa de vida e os determinantes de uma boa percepção de $\mathrm{QV}$, com o intuito de estimular os fatores positivos e intervir sobre os negativos.

$\mathrm{Na}$ presente pesquisa, observou-se que o domínio que considera em sua avaliação aspectos referentes às relações pessoais, ao apoio pessoal e à atividade sexual, foi determinante para a boa percepção de QV nos dois grupos de idosos. Na sequência de contribuição, apareceram o domínio do meio ambiente, o psicológico e o físico. Esta ordem de contribuição também foi encontrada nos estudos de Farenzena et al. (2007) e de Serbim e Figueiredo (2011). Situação diferente da encontrada no estudo de Pereira et al. (2006), o qual também investigou idosos jovens e longevos e constatou a seguinte ordem de contribuição geral: físico, meio ambiente, psicológico e relações sociais.

De acordo com Pereira et al. (2006), deve-se refletir sobre a ordem de contribuição dos domínios de avaliação da QV, no sentido de compreender a percepção global de QV de cada indivíduo. No julgamento que cada pessoa faz acerca de sua QV global, existem diferentes aspectos sendo considerados. Assim, os domínios de avaliação da QV contribuem para a percepção de vida em magnitudes diferentes, sendo que aqueles que se mostram mais influentes devem ser discutidos à luz da heterogeneidade existente entre os idosos, no que se refere ao sexo, à faixa etária, dentre outros aspectos.

Sobre o domínio psicológico, o qual considera em sua avaliação os sentimentos positivos e negativos, a autoestima, a espiritualidade, a imagem corporal e o pensar e aprender, e sobre o domínio do meio ambiente, o qual, por sua vez, considera a segurança física, os recursos financeiros, os cuidados com a saúde, o ambiente físico, o transporte, a informação, o ambiente no lar e as oportunidades de lazer (FLECK et al., 2008), pode-se afirmar que não houve diferenças significativas entre os grupos de idosos. Ambos alcançaram médias superiores a 60 nos escores destes domínios, indicando que os percebem positivamente em suas vidas e de maneira similar.

No contexto do domínio físico, esperava-se maior média entre os domínios da QV nos dois grupos de idosos, com base no estudo de Pereira et al. (2006), o qual investigou idosos jovens e longevos, encontrando médias mais elevadas nesse domínio, e também pelo fato destes indivíduos participarem de um programa de extensão universitária voltado à prática de exercícios físicos, e por integrarem efetivamente um programa de hidroginástica. Todavia, em ambos os grupos a média deste domínio ultrapassou o ponto médio da escala, sugerindo que os aspectos físicos também estão influenciando positivamente a avaliação da QV.

Fleck et al. (2008) informam que o domínio físico envolve a avaliação de vários aspectos que não apenas a satisfação com a mobilidade e o nível de energia, por exemplo. Fatores relacionados à dor e ao desconforto, ao nível de fadiga, ao sono e ao repouso, às atividades da vida cotidiana, à dependência de medicação, e à própria capacidade de trabalho também estão incluídos na avaliação deste domínio. Assim, as médias mais baixas encontradas em sua avaliação podem estar relacionadas à maior incidência de dores e desconfortos entre idosos, uma vez que, de acordo com Conte e Lopes (2005), estes indivíduos geralmente são mais suscetíveis às doenças características do processo de envelhecimento.

Apesar disso, os idosos longevos, ainda que em menor número que os jovens, continuam se mantendo ativos por meio da prática de hidroginástica no GETI. Este fato se mostra positivo no sentido de considerar a importância do exercício físico e da manutenção de um estilo de vida ativo 
nessa faixa etária que tende a apresentar mais problemas de saúde. A prática da hidroginástica pode contribuir para a capacidade funcional, saúde, bem-estar, socialização e autonomia de idosos de diferentes idades, e fica evidente que estes aspectos estão diretamente relacionados a uma boa percepção de QV (MAZO; CARDOSO; AGUILAR, 2006; CERRI; SIMÕES, 2007; TRIBESS; VIRTUOSO JÚNIOR; OLIVEIRA, 2012).

$\mathrm{Na}$ avaliação específica da QV, por sua vez, a faceta que avalia a capacidade de ter relações íntimas (INT) e a faceta que considera a participação em atividades do quotidiano, especialmente na comunidade (PSO), foram determinantes para a percepção positiva de vida dos idosos jovens. Em um estudo similar realizado por Maués et al. (2010), a PSO também teve maior impacto na percepção de QV dos idosos jovens, em comparação aos idosos longevos.

Segundo Freire (2000), na terceira idade, cujas características principais, em relação ao comportamento social, são a diminuição das capacidades sensoriais e a redução da prontidão para a resposta, outras habilidades podem ser especialmente importantes, tais como as de estabelecer e manter contato social, além de lidar com os comportamentos sociais decorrentes de preconceitos contra a velhice, geralmente expressos por meio de hesitação. Assim, acredita-se que a alta média encontrada nas facetas da INT e da PSO estejam relacionadas à participação ativa dos idosos na vida social, a qual pode estar se concretizando por meio do próprio envolvimento dos mesmos com as atividades do GETI, e contribuindo para os relacionamentos pessoais em diferentes perspectivas.

Já no grupo de idosos longevos, a faceta que se relaciona às preocupações, inquietações e temores acerca da morte e do morrer, foi percebida de forma mais positiva entre esses idosos, assim como constatado nos achados dos estudos de Serbim e Figueiredo (2011) e de Maués et al. (2010). Nesse contexto, é importante considerar o fenômeno da morte como inerente à vida em todas as suas formas. Acredita-se que por estarem em idades mais avançadas, os idosos longevos estão encarando a morte de forma mais natural e positiva que os idosos jovens, uma vez que a média da faceta MEM entre os idosos desse grupo $(82,3 \pm 17,2)$ ficou mais próxima de 100 (valor máximo) que a média alcançada na mesma faceta entre os idosos jovens $(66,5 \pm 22,6)$.

Além disso, entre os idosos longevos a faceta que se refere à independência na velhice e, portanto, descreve até que ponto se é capaz de viver autonomamente e tomar suas próprias decisões (AUT), apresentou média ligeiramente maior que a média dos idosos jovens. Apesar de não terem sido identificadas diferenças estatisticamente significativas entre os grupos, para os idosos longevos, a faceta da AUT ocupou o segundo lugar entre as facetas com maiores médias, enquanto para os idosos jovens, esta faceta ocupou o quarto lugar.

A manutenção da capacidade da autonomia no idoso permite que este tenha um envelhecimento saudável, mesmo que ele venha a ter qualquer tipo de doença crônica não transmissível. Esta capacidade, segundo Tahan e Carvalho (2010), está relacionada à satisfação com a vida, sendo muito valorizada por idosos. Nesse sentido, uma vez que os idosos longevos participam de um programa de hidroginástica e percebem sua vida de forma positiva, fica claro que estes indivíduos estão satisfeitos com sua autonomia e independência, tanto quanto os idosos mais jovens.

Em contrapartida, a análise estatística revelou uma diferença significativa entre a capacidade de se ter relações intímas (INT) de idosos jovens e longevos. No entanto, este resultado pode ser esperado, quando se considera que a maioria dos idosos longevos pertence ao sexo feminino e que o processo de envelhecimento é caracterizado pela predominância feminina, geralmente relacionada ao fatos das mulheres ficarem viúvas mais frenquentemente que os homens (CAMARANO; KANSO; MELLO, 2004). Apesar de os idosos longevos não estarem tão satisfeitos ou não estarem mantendo muitas relações de intimidade como os idosos jovens, a média do escore de percepção sobre esta faceta ultrapassou o ponto médio da escala $(55,2 \pm 9,2)$, e não impactou de forma significativa a boa percepção de QV como um todo.

Portanto, apesar de o grupo de idosos jovens ter apresentado maiores médias estatisticamente significativas nos domínios físico e das relações sociais e na faceta da intimidade da avaliação da QV, o grupo de idosos longevos apresentou uma percepção geral de QV tão boa quanto à percepção do primeiro grupo. 


\section{Considerações Finais}

Os idosos investigados no presente estudo apresentaram uma boa percepção de QV. Os idosos jovens apresentaram melhor percepção de QV nos domínios físico e das relações sociais e na faceta 'intimidade' que os longevos. Os idosos longevos, por sua vez, apresentaram melhor percepção de QV na faceta 'morte e morrer' quando comparados aos idosos jovens.

Acredita-se que a maior incidência de dores e desconfortos entre idosos, especialmente entre aqueles com idades mais avançadas, e a percepção menos positiva de alguns aspectos de avaliação do domínio físico (como o nível de fadiga, o sono e o repouso, entre outros), tenham sido determinantes para os resultados encontrados na avaliação deste domínio entre os idosos jovens e longevos. Na mesma perspectiva, acredita-se que a redução de vínculos e de relações sociais, característica do processo de envelhecimento, tenha contribuído para as médias mais baixas encontradas entre os idosos longevos no domínio das relações sociais e na faceta da intimidade, quando comparadas às médias encontradas neste domínio e nesta faceta no grupo de idosos jovens. Por fim, especula-se que a melhor percepção de QV na faceta 'morte e morrer' apresentada pelos idosos longevos, esteja relacionada à capacidade destes indivíduos de encarar a morte de forma mais natural, haja vista suas idades avançadas.

Os resultados deste estudo permitiram avançar no conhecimento relacionado à percepção de QV de idosos de distintas faixas de idade, especialmente entre aqueles com idades mais elevadas que, apesar de em menor número, integraram a amostra deste estudo. Além disso, contribuíram para alertar aos diferentes profissionais que trabalham com o público idoso acerca da importância das características e peculiaridades desse segmento populacional, assim como à percepção de QV dos idosos, com o intuito de estabelecer estratégias e buscar mecanismos que favoreçam o processo de envelhecimento saudável.

Uma vez que os benefícios da prática regular e sistematizada de exercícios físicos já estão bem documentados, percebe-se a premência em investigar as percepções, necessidades, desejos e limitações da vida de idosos no contexto das diferentes possibilidades de exercícios físicos, a fim de identificar as atividades potencialmente relacionadas a uma boa QV na velhice. É importante lembrar que a avaliação da QV depende das expectativas e percepções subjetivas de cada indivíduo, em estreita relação com o contexto no qual está inserido. Portanto, sugere-se que estudos futuros investiguem a percepção de QV de idosos em diferentes contextos, praticantes de outras modalidades de exercícios físicos que não apenas a hidroginástica, e com amostras maiores de idosos longevos.

\section{Referências}

ALVES, R. V. et al. Aptidão física relacionada à saúde de idosos: influência da hidroginástica. Revista Brasileira de Medicina do Esporte, São Paulo, v. 10, n. 1, p. 31-37, jan./fev. 2004.

BECKERT, M.; IRIGARAY, T. Q.; TRENTINI, C. M. Qualidade de vida, cognição e desempenho nas funções executivas de idosos. Estudos de Psicologia, Campinas, v. 29, n. 2, p. 155-162, abr./jun. 2012.

CAMARANO, A. A.; KANSO, S.; MELLO, J. L. Como vive o idoso brasileiro. In: CAMARANO, A. A. (Org.). Os novos idosos brasileiros: muito além dos 60? Rio de Janeiro: IPEA, 2004. p. 2573.

CERRI, A.; SIMÕES, R. Hidroginástica e idosos: por que eles praticam? Movimento, Porto Alegre, v. 13, n. 1, p. 81-92, jan./abr. 2007. 
CONTE, E. M. T.; LOPES, A. S. Qualidade de vida e atividade física em mulheres idosas. Revista Brasileira de Ciências do Envelhecimento Humano, Passo Fundo, v. 2, n. 1, p. 61-75, jan./jun. 2005.

FARENZENA, W. P. et al. Qualidade de vida em um grupo de idosos de Veranópolis. Revista Kairós, São Paulo, v. 10, n. 2, p. 225-243, dez. 2007.

FLECK, M. P. A.; CHACHAMOVICH, E.; TRENTINI, C. Development and validation of the Portuguese version of WHOQOL-OLD. Revista de Saúde Pública, São Paulo, v. 40, n. 5, p. 785791 , oct. 2006.

FLECK, M. P. A. et al. A avaliação de qualidade de vida: guia para profissionais da saúde. Porto Alegre: Artmed, 2008.

FLECK, M. P. A. et al. Aplicação da versão em português do instrumento abreviado de avaliação da qualidade de vida 'WHOQOL-bref'. Revista de Saúde Pública, São Paulo, v. 34, n. 2, p. 178183, abr. 2000.

FREIRE, S. Envelhecimento bem-sucedido e bem estar psicológico. In: NERI, A. L.; FREIRE, A.S. (Orgs.). E por falar em boa velhice. Campinas: Papirus, 2000. p. 21-31.

IBGE - INSTITUTO BRASILEIRO DE GEOGRAFIA E ESTATÍSTICA. Censo demográfico 2010: características da população e dos domicílios - resultados do universo. Rio de Janeiro: IBGE, 2011.

IBGE. Síntese de indicadores sociais: uma análise das condições de vida da população brasileira 2010. Rio de Janeiro: IBGE, 2010.

IBGE. Projeção da população do Brasil por sexo e idade - 1980-2050 - revisão 2008. Rio de Janeiro: IBGE, 2008.

IPEA - INSTITUTO DE PESQUISA ECONÔMICA APLICADA. Disponível em: <www.ipea.gov.br>. Acesso em: 31 out. 2012.

JOIA, L. C.; RUIZ, T.; DONALISIO, M. R. Life satisfaction among elderly population in the city of Botucatu, Southern Brazil. Revista de Saúde Pública, São Paulo, v. 41, n. 1, p. 131-138, fev. 2007.

MAUÉS, C. R. et al. Avaliação da qualidade de vida: comparação entre idosos jovens e muito idosos. Revista Brasileira de Clínica Médica, São Paulo, v. 8, n. 5, p. 405-410, set./out. 2010.

MAZO, G. Z. Atividade física, qualidade de vida e envelhecimento. Porto Alegre: Meridional, 2008.

MAZO, G. Z.; CARDOSO, F. L.; AGUIAR, D. L. Programa de hidroginástica para idosos: motivação, auto-estima e autoimagem. Revista Brasileira de Cineantropometria e Desempenho Humano, Florianópolis, v. 8, n. 6, p. 67-72, 2006.

MAZO, G. Z.; LOPES, M. A.; BENEDETTI, T. B. Atividade física e o idoso: concepção gerontológica. 3. ed. rev. aum. Porto Alegre: Sulina, 2009. 
OMS - ORGANIZAÇÂO MUNDIAL DA SAÙDE. Envelhecimento ativo: uma política de saúde. Brasília: Organização Pan-Americana da Saúde, 2005.

PEREIRA, R. J. et al. Contribuição dos domínios físico, social, psicológico e ambiental para a qualidade de vida global de idosos. Revista de Psiquiatria do Rio Grande do Sul, Porto Alegre, v. 26, n. 1, p. 27-38, jan./abr. 2006.

SERBIM, A. K., FIGUEIREDO, A. E. P. L. Qualidade de vida de idosos num grupo de convivência. Scientia Médica, Porto Alegre, v. 21, n. 4, p. 166-172, out. 2011.

SIMÕES, R. R.; PORTES JÚNIOR, M. MOREIRA, W. W. Idosos e hidroginástica: corporeidade e vida. Revista Brasileira de Ciência e Movimento, Taraguatinga, v. 19, n. 4, p. 40-50, set./dez. 2011.

TAHAN, J.; CARVALHO, A. C. D. de. Reflexões de idosos participantes de grupos de promoção de saúde acerca do envelhecimento e da qualidade de vida. Saúde e Sociedade, São Paulo, v. 19, n. 4, p. $878-888$, out./dez. 2010.

TOSCANO, J. J. O.; OLIVEIRA, A. C. C. Qualidade de vida em idosos com distintos níveis de atividade física. Revista Brasileira de Medicina do Esporte, São Paulo, v. 15, n. 3, p. 169-173, maio/jun. 2009.

TRIBESS, S.; VIRTUOSO JÚNIOR, J. S.; OLIVEIRA, R. J. Atividade física como preditor da ausência de fragilidade em idosos. Revista da Associação Médica Brasileira, São Paulo, v. 58, n. 3, p. 341-347, maio/jun. 2012.

UENO, D. T. et al. Efeitos de três modalidades de atividade física na capacidade funcional de idosos. Revista Brasileira de Educação Física e Esporte, São Paulo, v. 26, n. 2, p. 273-281, abri./jun. 2012.

WHOQOL GROUP. Development of the World Health Organization WHOQOL-BREF Quality of Life Assessment. Psychological Medicine, Cambridge, v. 28, n. 3, p. 551-558, maio 1998.

WHOQOL GROUP. The World Health Organization Quality of Life Assessment (WHOQOL): position paper from the World Health Organization. Social Science and Medicine, Oxford, v. 41, n. 10, p. 1403-1409, nov. 1995. 